\title{
Electron correlation and charge transfer at the Ni/Co interface
}

\author{
S. S. Dhesi, ${ }^{a}{ }^{2}$ E. Dudzik, H. A. Dürr, and G. van der Laan \\ Magnetic Spectroscopy Group, Daresbury Laboratory, Warrington WA4 4AD, United Kingdom \\ N. B. Brookes \\ ESRF, BP 220, F-38043 Grenoble, France
}

\begin{abstract}
The evolving magnetism and electronic structure at the Ni/Co interface have been studied using $\mathrm{x}$-ray absorption spectroscopy (XAS) and x-ray photoemission spectroscopy (XPS) with circularly polarized $\mathrm{x}$ rays. Deposition of ultrathin Ni films on thin films of Co grown on $\mathrm{Cu}(001)$ results in an intensity enhancement across the Co $L_{2.3}$ absorption edge. By comparison, the intensity of the Ni $L_{2.3}$ edge decreases as a function of Ni film thickness. The relative changes in the $\mathrm{Ni}$ and Co XAS intensities are interpreted as an electronic charge transfer from the Co to the Ni. Distinct changes in the Co $2 p$ XAS and XPS line shapes after addition of the Ni overlayer imply a modification of the Co $3 d$ electron correlation due to the charge transfer. The change in the electronic structure is related to the interface magnetism using magnetic circular dichroism sum rule analysis. (C) 2000 American Institute of Physics. [S0021-8979(00)65308-2]
\end{abstract}

Recent developments in thin film growth technology have enabled the fabrication of novel materials with high potential for commercial application in the magnetic recording industry. In parallel with these advances, thin films have been investigated with the aim of understanding the changing electronic and magnetic structures as the film thickness is varied. ${ }^{1-3} \mathrm{X}$-ray absorption spectroscopy (XAS) and x-ray photoelectron spectroscopy (XPS) combined with circularly polarized synchrotron radiation are powerful techniques with which to probe the surfaces and interfaces of these thin films. XAS using circularly polarized radiation gives rise to $\mathrm{x}$-ray magnetic circular dichroism (XMCD) which yields elementspecific spin and orbital magnetic moments. ${ }^{4,5}$ On the other hand, magnetic circular dichroism in photoemission (MCDPE) is a highly surface sensitive tool which can be used to determine the surface dependence of the orbital moment. ${ }^{6}$

The line shapes of isotropic and dichroic spectra can change dramatically for varying film thickness due to electron transfer and hybridization at the interface or due to $3 d$ electron correlation effects. Srivastava et al. ${ }^{3}$ have shown that the thickness dependent density of $3 d$ holes for Ni thin films grown on $\mathrm{Cu}(001)$ gives rise to a modified XAS line shape. Dürr et ll $^{7}$ have used changes in XAS and XPS line shapes to correlate the electron localization with the magnetic ordering of ultrathin Mn films.

In the present study, charge redistribution at the $\mathrm{Ni} / \mathrm{Co}$ interface and the effects of an evolving electronic structure on interface magnetism are investigated using core-level spectroscopies. The deposition of Ni onto a 4 ML Co film results in distinct changes in the Co $2 p$ XAS and XPS line shapes due to modifications in the $d$ electron correlation.

$\mathrm{The} \mathrm{Cu}(001)$ substrate was prepared by repeated cycles of $\mathrm{Ar}^{+}$ion bombardment and annealing to $700 \mathrm{~K}$ in ultrahigh vacuum (UHV). Low-energy electron diffraction and XPS

\footnotetext{
a) Author to whom correspondence should be addressed; electronic mail: s.s.dhesi@dl.ac.uk
}

indicated a highly ordered surface without $\mathrm{C}$ and $\mathrm{O}$ contamination. A 4 ML Co film was subsequently grown onto the $\mathrm{Cu}(001)$ at temperatures between 270 and $300 \mathrm{~K}$ followed by deposition of $\mathrm{Ni}$ at similar temperatures. The core-level measurements were done using $85 \%$ circularly polarized light at beamline ID12B of the European Synchrotron Radiation Facility (ESRF) in Grenoble. XPS spectra were recorded using a hemisperical analyzer in a normal emission geometry with the light incident at $60^{\circ}$ to the surface normal. XAS spectra were recorded in total yield mode with the light incident at $45^{\circ}$. The XAS spectra were normalized to a constant edge jump to yield information on a per atom basis. Saturation effects are negligible for the geometry and thin films used in this study. ${ }^{8}$ The magnetic dipole term in the spin sum rule is negligible in the present study since the data were taken near the magic angle. ${ }^{9}$ All other experimental details are given elsewhere. $^{10}$

Figure 1 shows Co $L_{2.3}$ XAS spectra for a 4 ML Co film before (dashed line) and after (drawn line) the addition of 1 ML of Ni. The spectra show features due to electric-dipole transitions from the spin-orbit split $2 p$ core levels to unoccupied $s$ and $d$ states. The addition of the Ni overlayer results in increased intensity across both edges and in the appearance of additional satellite features, marked by the arrows. The inset of Fig. 1 shows the Co $L_{2.3}$ integrated intensity for varying Ni coverages after background subtraction which removes the contributions due to transitions into the unoccupied $s$ and continuum states. In order to show the rise in the integrated intensity per $\mathrm{Ni} / \mathrm{Co}$ interface site, $\Delta\left(A L_{3}+A L_{2}\right)$, the raw change in the integrated intensities was divided by the $\mathrm{Ni}$ film thickness. Without this procedure $\Delta\left(A L_{3}\right.$ $+A L_{2}$ ) would yield the average number of $d$ holes for all Co coordinations in the film. The increase in the integrated intensity over the Co $L_{2.3}$ edge in Fig. 1 reflects the increase in the number of Co $d$ holes $\left(n_{h}\right)$. Similar XAS spectra recorded for the $\mathrm{Ni} L_{2.3}$ edge showed that the number of $\mathrm{Ni} d$ holes decreases with decreasing Ni thickness. ${ }^{10}$ The dramatic increase in $n_{h}$ (cf. inset of Fig. 1) after the Ni deposition 


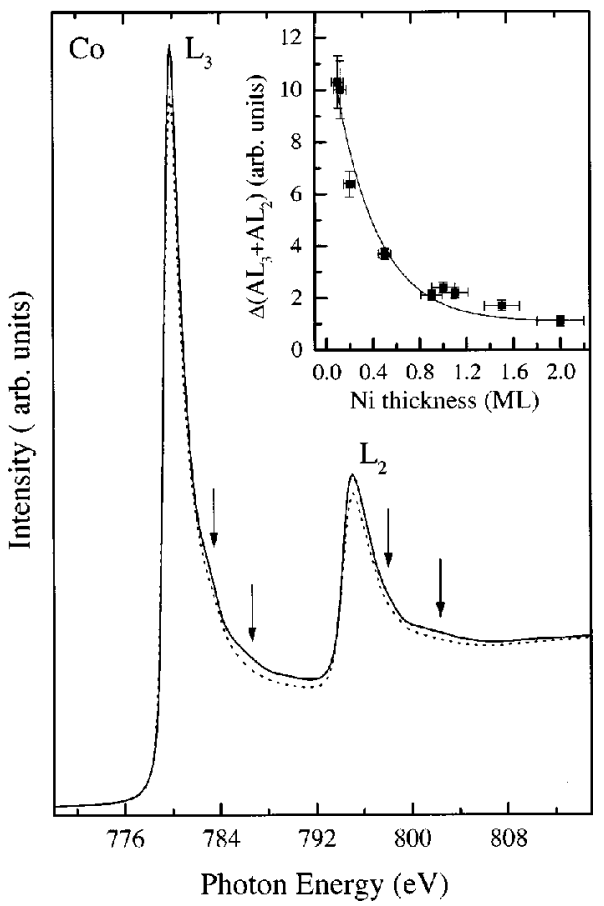

FIG. 1. Co $L_{23}$ XAS spectra for a $4 \mathrm{ML}$ film before (dashed line) and after (drawn line) the addition of $1 \mathrm{ML}$ of Ni normalized to a constant edge jump. The inset shows the increase in the background subtracted integrated intensity over the $\mathrm{Co}_{23}$ edges as a function of Ni coverage. The drawn line is a guide to the eye.

together with the decrease in the Ni $d$ holes suggests a transfer of charge at the interface from Co to Ni. The removal of charge from the Co sites after the addition of the Ni ML also influences the $d$ electron correlation which results in the appearance of the satellite features marked by the arrows in Fig. 1.

Figure 2(a) shows Co $L_{2.3} \mathrm{XMCD}$ spectra recorded for a 4 ML film before (dashed line) and after (drawn line) the deposition of $1 \mathrm{ML}$ of Ni. The increase in the XMCD intensity in Fig. 2(a) clearly shows an increase in the Co spin moment. Sum rule analysis gives an increase in the Co spin moment from $1.4 \pm 0.1 \mu_{B}$ to $1.9 \pm 0.1 \mu_{B}$. The error bars account for approximations used in the derivation of the sum rules ${ }^{11}$ and systematic errors in the experimental data.

Figure 2(b) shows the XMCD spectra recorded for a 0.2 ML (dashed line) and 2 ML (drawn line) Ni film grown on a 4 ML Co film. The inset shows the integrated $L_{3}$ edge dichroism $\left(\Delta L_{3}\right)$ as a function of Ni film thickness. The increasing transfer of electronic charge per interface atom for decreasing Ni film thickness (cf. inset of Fig. 1) is seen to be correlated with a decrease in the Ni $L_{3}$ dichroism [cf. inset of Fig. 2(b)]. Sum rule analysis of the XMCD spectra in Fig. 2(b) gives a $\mathrm{Ni}$ spin moment of $0.4 \pm 0.06 \mu_{B}$ and 0.22 $\pm 0.06 \mu_{B}$ for the 2 and 0.2 ML films, respectively. The thickness dependent number of $\mathrm{Ni} d$ holes has been included in the calculation of the spin moments. ${ }^{3,10}$

The transfer of charge from Co to Ni therefore increases the Co spin moment and reduces the Ni spin moment. In a one-electron model the charge redistribution will affect the exchange-split minority and majority bands and result in a modified magnetic structure at the interface. The precise
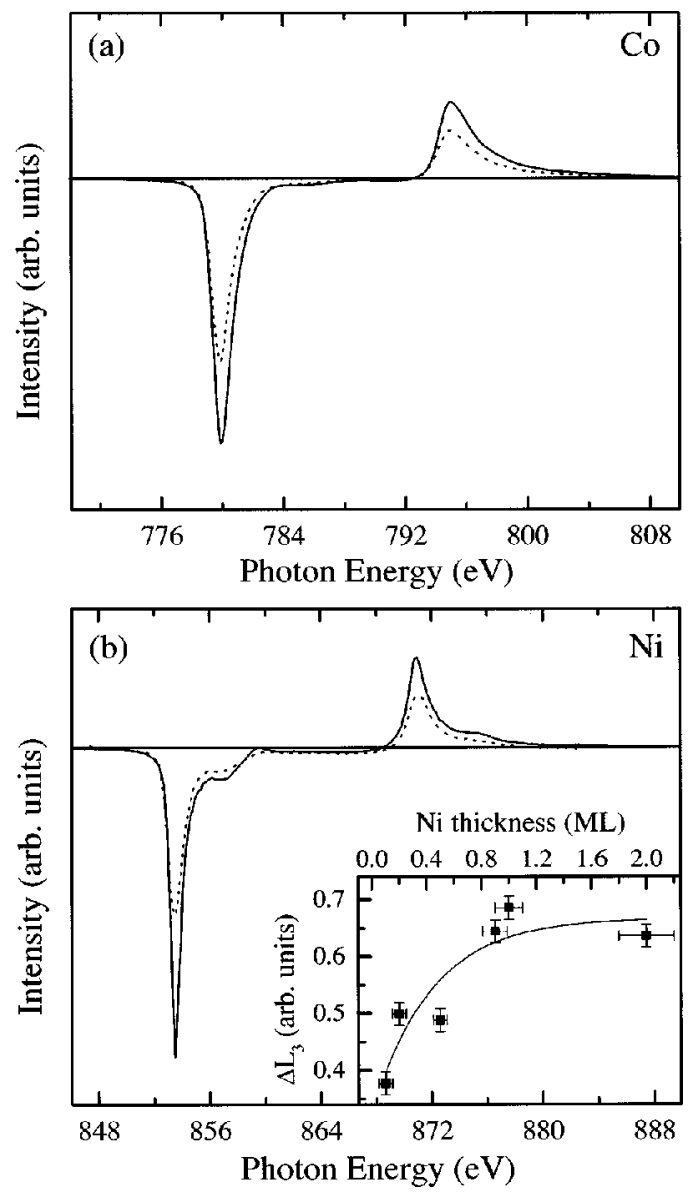

FIG. 2. (a) XMCD spectra for a 4 ML Co film before (dashed line) and after (drawn line) the addition of $1 \mathrm{ML}$ of Ni. (b) XMCD spectra for $0.2 \mathrm{ML}$ (dashed line) and 2 ML (drawn line) Ni films deposited on 4 ML of Co grown on $\mathrm{Cu}(001)$. The inset shows the change in the integrated area of the $L_{3}$ dichroism as a function of $\mathrm{Ni}$ film thickness. The drawn line is a guide to the eye.

mechanism of this process requires detailed first-principles calculations which reproduce the charge redistribution at the interface. However, our Korringa-Kohn-Rostoker Green's function calculations invoking the local-spin-density approximation (LSDA) show no change in the $d$ occupancy of the $\mathrm{Ni}$ or $\mathrm{Co}$ at the Ni/Co interface. Furthermore, these calculations also predict bulk values for the $\mathrm{Ni}$ and $\mathrm{Co}$ spin moments at the interface in agreement with previously reported calculations. ${ }^{12}$ The discrepancy between the experimental and theoretical results is most likely due to the limitations of the LSDA in accurately determining the spin dependent exchange-correlation energy for the ferromagnetic interface.

In a configuration interaction (CI) model, the origin of ferromagnetism in $\mathrm{Ni}$ metal has been explained in terms of a small $d^{8}$ weight in the ground state. ${ }^{13,14}$ The transfer of charge will affect the $d$ weights and therefore the spin moments. However, the application of the CI model to other transition metals is more complicated because of the increasing number of configurations involved. For the Ni, thickness dependent XAS and XMCD line shape changes show that the charge redistribution reduces the $d^{8}$ weight and increases the diffuse magnetism arising from delocalized $s p$ states. ${ }^{10}$ In 


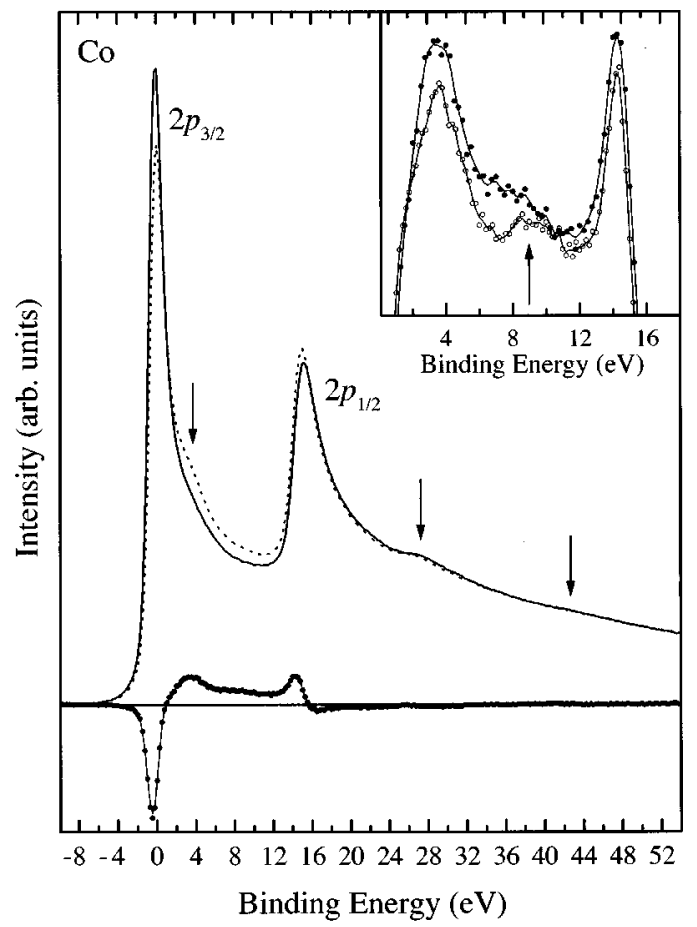

FIG. 3. Co $2 p$ XPS spectra for a 4 ML film with photon helicity parallel (dashed line) and antiparallel (drawn line) to the sample magnetization. Satellite features are indicated by the downwards pointing arrows. The resulting MCDPE is shown below (solid circles; the line drawn through the MCDPE is a Fourier smoothing of the data). The inset shows details of the Co MCDPE (solid circles) between the $2 p_{3 / 2}$ and $2 p_{1 / 2}$ peaks together with an MCDPE recorded for a $4 \mathrm{ML}$ Co film after deposition of Ni (open circles). An additional feature appears after the deposition of the $\mathrm{Ni}$ and is marked by the upwards pointing arrow.

terms of the CI model, these effects are then mainly responsible for the decreasing Ni spin moment.

Figure 3 shows Co $2 p$ XPS spectra from a 4 ML Co film recorded with the light polarization parallel (dashed line) and antiparallel (drawn line) to the sample magnetization direction. The core hole spin-orbit interaction divides the spectrum into $2 p_{3 / 2}$ and $2 p_{1 / 2}$ structures, separated by $15.1 \mathrm{eV}$. In addition, satellite features at 3.7, 27.2, and $42.7 \mathrm{eV}$ above the $2 p_{3 / 2}$ peak are indicated by downward pointing arrows. These satellites have been discussed previously, ${ }^{15,16}$ with the $3.7 \mathrm{eV}$ feature being ascribed to a two-hole final state in an analogous manner to the case for Ni XPS satellites. ${ }^{14}$ The MCDPE spectrum, which is the difference between the two displayed XPS spectra, is also shown in Fig. 3 (solid circles with a drawn line to guide the eye). The Co $2 p_{3 / 2}$ MCDPE exhibits a sharp negative peak followed by a broad positive structure, while the $2 p_{1 / 2}$ edges display a weaker structure reversed in sign. This line shape is similar to that reported by Dürr et al. ${ }^{17}$ for $\mathrm{Fe}$ thin films grown on $\mathrm{Cu}(001)$ which could not be reproduced by one-electron calculations. From the similarity between the Fe MCDPE ${ }^{15,17}$ and the Co MCDPE presented in this work it can be expected that a one-electron approach will also fail to reproduce the Co MCDPE. For the latter this is in part due to the electron correlation features in the MCDPE which are not considered in a one-electron approach. The dichroism arises due to the interaction of the magnetically aligned valence band spin moments with the orbital moment of the core hole. A change in the valence band spin moments after the deposition of Ni can therefore affect the MCDPE line shape.

The inset of Fig. 3 shows details of the MCDPE spectrum between the $2 p_{3 / 2}$ and the $2 p_{1 / 2}$ peaks together with the MCDPE spectrum for the same Co film after the addition of $\mathrm{Ni}$ (open circles). The spectra have been normalized to the $2 p_{3 / 2}$ MCDPE intensity minimum. The change in the line shape of the lower binding energy peak and the appearance of an extra feature (indicated by the upward pointing arrow) is evident. The electron transfer from Co to the $\mathrm{Ni}$, established by XAS, is therefore also evident in the MCDPE. The removal of $d$ electrons from the Co alters the Co valence electron correlation which in turn affects the intensity of the satellite features. ${ }^{14}$

The magnetism and electronic structure of the $\mathrm{Ni} / \mathrm{Co}$ interface has been studied using core-level spectroscopy of ultrathin Ni films deposited on a $4 \mathrm{ML}$ Co film. XAS and XMCD indicate a charge transfer from the Co to the $\mathrm{Ni}$, during interface formation, which results in an increase in the Co spin moment and a reduction of the Ni spin moment. The line shapes of the Co XAS and XPS spectra are shown to be sensitive to the $d$ occupation and may be used to probe electron correlation effects in thin film interfaces induced by charge redistribution.

This research was supported under EPSRC Grant No. GR/L38240. The authors thank K. Larsson for his help and technical assistance.

${ }^{1}$ D. Weller, J. Stöhr, R. Nakajima, A. Carl, M. G. Samant, C. Chappert, R. Mégy, P. Beauvillain, P. Veillet, and G. A. Held, Phys. Rev. Lett. 75, 3752 (1995).

${ }^{2}$ J. H. Dunn, D. Arvanitis, and N. Mårtensson, Phys. Rev. B 54, R11157 (1996).

${ }^{3}$ P. Srivastava, N. Haack, H. Wende, R. Chauvistré, and K. Baberschke, Phys. Rev. B 56, R4398 (1997).

${ }^{4}$ B. T. Thole, P. Carra, F. Sette, and G. van der Laan, Phys. Rev. Lett. 68, 1943 (1992).

${ }^{5}$ P. Carra, B. T. Thole, M. Altarelli, and X. Wang, Phys. Rev. Lett. 69, 2307 (1993).

${ }^{6}$ G. van der Laan, M. A. Hoyland, M. Surman, C. F. J. Flipse, and B. T. Thole, Phys. Rev. Lett. 69, 3827 (1992).

${ }^{7}$ H. A. Dürr, G. van der Laan, D. Spanke, F. U. Hillebrecht, and N. B. Brookes, Phys. Rev. B 56, 8156 (1997).

${ }^{8}$ R. Nakajima, J. Stohr, and Y. U. Idzerda, Phys. Rev. B 59, 6421 (1999).

${ }^{9}$ G. van der Laan, Phys. Rev. B 57, 5250 (1998).

${ }^{10}$ S. S. Dhesi, E. Dudzik, H. A. Dürr, G. van der Laan, and N. B. Brookes, Phys. Rev. B 60, 12852 (1999).

${ }^{11}$ R. Wu and A. J. Freeman, Phys. Rev. Lett. 73, 1994 (1994).

${ }^{12}$ A. M. N. Niklasson, B. Johansson, and H. L. Skriver, Phys. Rev. B 59, 6373 (1999).

${ }^{13}$ T. Jo and G. A. Sawatzky, Phys. Rev. B 43, 877 (1991).

${ }^{14}$ G. van der Laan and B. T. Thole, J. Phys.: Condens. Matter 4, 4181 (1992).

${ }^{15}$ C. M. Schneider, U. Pracht, W. Kuch, A. Chassé, and J. Kirschner, Phys. Rev. B 54, R15618 (1996).

${ }^{16}$ S. Raaen, Solid State Commun. 60, 991 (1986).

${ }^{17}$ H. A. Dürr, G. van der Laan, D. Spanke, F. U. Hillebrecht, and N. B. Brookes, Europhys. Lett. 40, 171 (1997). 